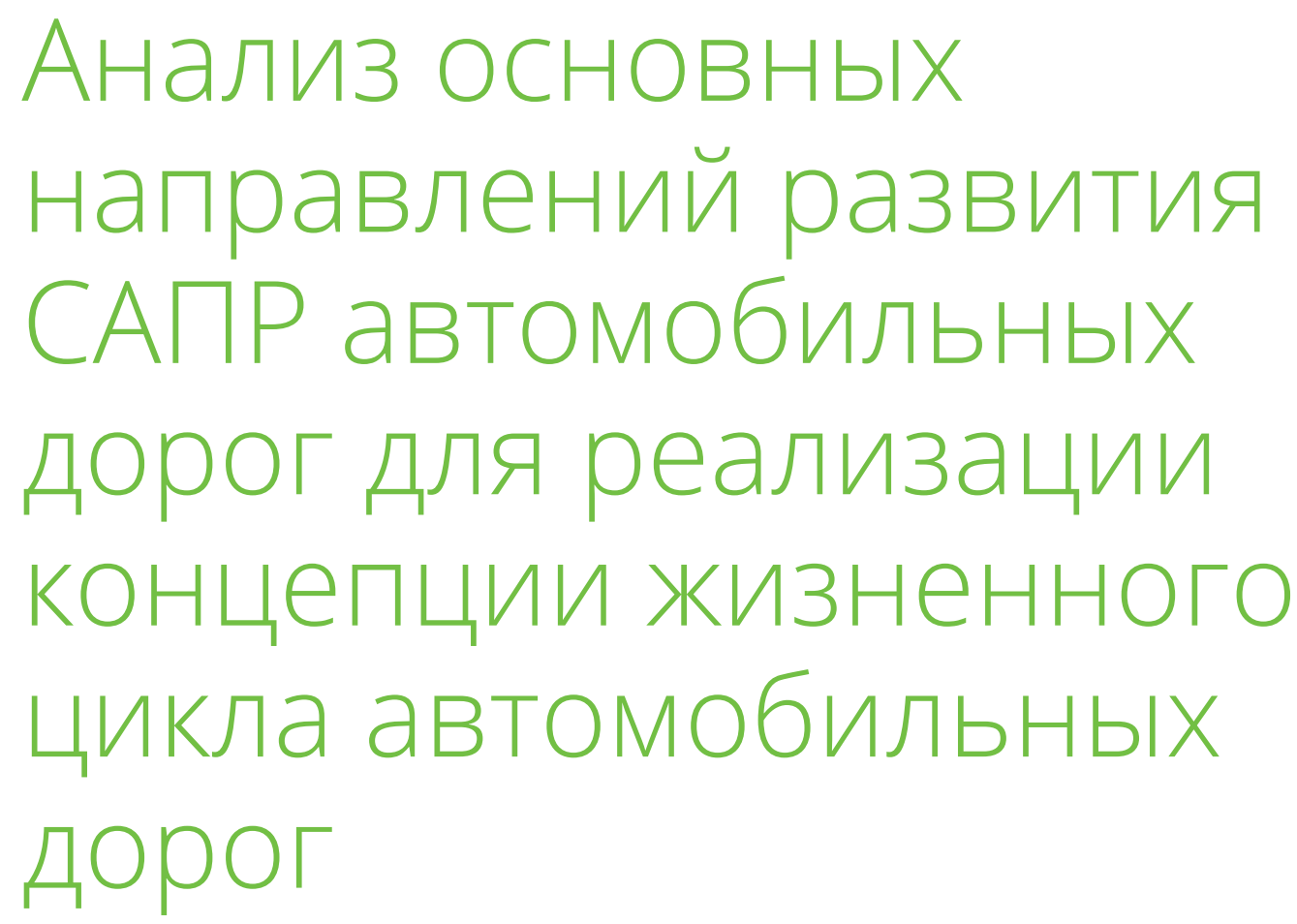

Миронюк В.П., Д.э.н., профессор РГСУ (г. Ростов-на-Дону), гИП, старший научный сотрудник Северо-Кавказского филиала ОАО «ГИПРОДОРНИИ»

Фиалкин В.В., ассистент РГСУ (г. Ростов-на-Дону)

Проводится анализ САПР, представленных на российском рынке, в рамках формирования информационной модели объектов строительства на базе ВІМ-технологии. Описаны основные этапы и взаимосвязи использования программных продуктов для реализации ВІМ-технологии. Определены основные направления дальнейшего развития отечественных САПР для реализации концепции поддержки жизненного циикла дороги.

Реализация подхода на основе «информационного моделирования дороги» [1] (далее ИМД) заставляет учитывать все её особенности как линейного объекта, обращая особое внимание на различия линейных объектов (ЛО) и объектов капитального строительства (ОКС). Реализация поддержки концепции жизненного цикла на базе ИМД получила своё развитие благодаря эволюции САПР и ГИС автомобильных дорог. Сегодня на рынке САПР автомобильных дорог основными являются комплексы следующих компаний: Autodesk (США), CREDO (Белоруссия), «ИндорСофт» (г. Томск), «Топоматик» (г. СанктПетербург). Кроме этого, существует достаточно большое количество продуктов, которые были разработаны для решения узких задач проектирования автомобильных дорог, например: продукты компании PTV (Visum, Vissim), программные комплексы Aimsun, FORUM8, KorFin, CARD/1 и другие. Некоторые продукты разработаны и ис- пользуются в крупных проектных организациях, к ним можно отнести GIP-M - программный комплекс, разработанный и использующийся в ОАО «ГИПРОДОРНИИ». Но не все присутствующие на рынке программные комплексы, во-первых, учитывают специфику проектирования ЛО, во-вторых, охватывают весь спектр задач, которые стоят перед проектировщиками для создания ИМД.

При формировании информационной модели объектов инфраструктуры автомобильных дорог необходимо определиться с основными функциями, которые присущи тому или иному этапу жизненного цикла автомобильной дороги. В первую очередь нас интересует этап проектирования. Другие этапы жизненного цикла, такие как строительство, эксплуатация и использование за пределами расчётного эксплуатационного цикла должны стать предметом дальнейшего исследования. 
В общем виде ВIM-технология основана на 3D-модели строительных объектов, которая связана с информационной базой данных, где каждому элементу модели присвоены дополнительные атрибуты, такие как материал, его свойства, цена и т.д. Другими словами, информационная модель зданий и сооружений.

Основные функции, которые должны быть реализованы на стадии проектирования, включают в себя три блока:

1. Оценка функционирования объекта и предварительная стоимость различных вариантов на предпроектной стадии.

2. Визуализация проектных решений для проведения роад-шоу, в том числе для привлечения инвесторов.

3. Внедрение принципов проектного менеджмента на стадии подготовки проекта.

Проектирование автомобильной дороги как процесс, включает в себя три фазы: подготовка и согласование проекта планировки и межевания, подготовка и согласование проектной документации и подготовка рабочей документации. Каждой фазе характерны свои особенности, которые на других стадиях не встречаются.

Как уже было сказано выше, при разработке проекта планировки необходимо принимать решение по местоположению будущей трассы. На этой стадии нужно разработать несколько вариантов прохождения автомобильной дороги с определением её основных технико-экономических показателей, к которым можно отнести длину трассы, количество полос, количество искусственных сооружений и их основные технико-экономические характеристики, укрупнённую стоимость и т.д. Данные технические показатели должны обеспечить существующую и перспективную интенсивность дорожного движения, учитывать всю зону влияния проектируемой автомобильной дороги, обеспечивать безопасность дорожного движения. Это возможно выполнить при помощи программного обеспечения, которое реализует математическое моделирование транспортных потоков. К известным комплексам, которые предназначены для решения такого рода задач, можно отнести: Visum, Vissim, Aimsun и FORUM8. Программный комплекс KorFin предназначен для быстрого эскизного вы- полнения проектов на стадии подготовки к тендерам для демонстрации вариантов и расчёта предварительной стоимости различных вариантов, но не оценивает функциональные возможности будущего объекта с точки зрения пропускной способности.

На стадии проектной документации, необходимо проведение мероприятий по внедрению принципов проектного менеджмента, что позволит значительно повысить качество разрабатываемой проектной документации, и в конечном итоге позволит значительно сократить временные, финансовые и трудовые ресурсы. Внедрение принципов проектного менеджмента касается проверки отдельных разделов проекта, которые выполняются различными специалистами, структурными подразделениями или даже проектными институтами, их взаимной увязки, соблюдения порядка разработки проекта, расчёта отдельных его элементов и узлов. Реализация данной функции возможна только в рамках формирования информационной модели на базе ВIM-технологии.

В общем виде BIM-технология основана на 3D-модели строительных объектов, которая связана с информационной базой данных, где каждому элементу модели присвоены дополнительные атрибуты, такие как материал, его свойства, цена и т.д. Другими словами, информационная модель зданий и сооружений.

В отличие от обычных программ 2D, программа 3D-проектирования моделей на основе BIM-технологии в строительстве хранит не просто графические объекты, а информацию, позволяющую автоматически создавать чертежи и отчёты, выполнять анализ проекта, моделировать график выполнения работ, эксплуатацию объектов и т.д.

Также программа 3D-проектирования на основе технологии информационного моделирования позволяет поддерживать распределённые группы, благодаря чему несколько пользователей (проектировщик, подряд- чик, эксплуатирующая организация) могут работать с единой моделью на протяжении всего жизненного цикла объекта, что исключает избыточность, повторный ввод и потерю данных, ошибки при их передаче и преобразовании.

Реализация информационной модели объекта, как правило, строится на нескольких программных продуктах и их количество зависит от назначения самого объекта, его размера, поставленных задач и т.д. Наилучший вариант, когда продукты разработаны одной компанией или изначально предусматривалось совместное использование ПО различных производителей.

Рассмотрим на примере продуктов компании Autodesk использование BIM-технологии. В настоящее время программы этой компании позволяют наиболее полно и широко реализовать данный подход к ОКС. Программные продукты компании Autodesk позволяют осуществлять поддержку информационной модели объекта, но в основном предназначены только для ОКС, не учитывая специфические особенности автомобильных дорог. Хотя в линейке Autodesk есть модуль AutoCAD Civil 3D, который позиционируется как продукт, позволяющий ускорить процесс разработки высококачественных проектов в сфере транспорта, землеустройства и инфраструктуры. Тем не менее, данный продукт не учитывает многие особенности проектирования автомобильных дорог.

В последнее время технология BIM достаточно бурно развивается, обсуждается и представлена многими публикациями в интернете и других открытых источниках, но до настоящего момента нет чёткого понимания, какие продукты, в какой последовательности и для каких задач использовать. Как правило, на семинарах рассказывается о возможностях отдельных продуктов для решения той или иной задачи. Единого, комплексного и логически завершённого представления на примере одного проекта, к сожале- 
нию, увидеть не удалось. Для определения перечня и последовательности использования продуктов компании Autodesk в первую очередь сформулируем задачи, которые необходимо решить в процессе подготовки проектной документации.

Задачи, которые стоят перед проектировщиками, можно разбить на две большие группы.

1. Проверка проектных решений на предпроектной стадии:

- быстрая разработка концептуального проекта с визуализацией;

- оценка применения тех или иных принципиальных конструктивных решений;

- определение предварительных технико-экономических показателей для каждого из вариантов.

2. Проверка проектных решений на проектной стадии и стадии создания рабочей документации:

- определение оптимальных конструктивных схем, узлов, оборудования и материалов;

- проверка и взаимная увязка различных разделов проекта между собой;

- определение приведённых затрат с учётом срока эксплуатации объекта, что позволит обосновать применение инновационных материалов, технологий, методов строительства и т.д.;

- подготовка визуализации проекта для проведения роад-шоу, в том числе для привлечения инвестиций.

Для реализации указанных задач предлагается использовать следующие программные продукты компании Autodesk:

1. Программный комплекс Autodesk Building Design Suite версии Ultimate, который включает в себя, в том числе: - AutoCAD - система двух- и трёхмерного автоматизированного проектирования и подготовки документации. Пользователи AutoCAD могут адаптировать продукт для решения конкретных задач.

- AutoCAD Structural Detailing - программа проектирования строительных конструкций, предоставляющая широкий набор инструментов и знакомую среду AutoCAD. Интуитивные функции делают простым процесс деталировки и выпуска рабочих чертежей стальных и железобетонных строительных конструкций.
- Autodesk 3ds Max Design - система подготовки наглядных изображений и видеороликов, обладающих профессиональным качеством, для демонстрации проектных идей.

autodesk Navisworks Manage инструмент интеграции данных из различных источников для комплексной проверки проектов, координации и количественного анализа.

- Autodesk Revit - инструменты на базе технологии BIM для архитектурного проектирования, проектирования инженерных систем и строительных конструкций, а также моделирования строительства. В состав комплекса входят следующие продукты:

1. Revit Architecture. Программное обеспечение разработано для архитекторов и предназначено для полной свободы творчества при проектировании, улучшенной координации информации и эффективного представления готовых проектов.

2. Revit Structure. Программное обеспечение, предназначенное для проектирования и предварительного анализа строительных конструкций.

3. Revit MEP. Система автоматизированного проектирования в зданиях инженерных систем любой сложности. САПР Autodesk Revit MEP адресована проектировщикам отопительных, вентиляционных, электрических и санитарно-технических систем и основана на технологии информационного моделирования зданий (BIM).

autodesk Inventor - 3D-САПР для проектирования оборудования, которым оснащаются здания. Это удобная в работе 3D-САПР для проектирования деталей, изделий и выпуска чертежей.

2. Дополнительно необходим Autodesk Civil 3D - профессиональное решение для гражданского строительства, основанное на технологии BIM, которое позволяет ускорить процесс разработки высококачественных проектов в сфере транспорта, землеустройства и инфраструктуры. Кроме этого, Autodesk Civil 3D включает средства для геопространственного анализа с целью выбора подходящей строительной площадки, анализа ливневых стоков, составления сметы и динамического расчёта объёмов земля- ных работ для оптимизации расходов материалов.

3. Программное обеспечение Autodesk Vault представляет собой удобную систему управления файлами проекта. Используя возможности Autodesk Vault 2014, разработчики отслеживают изменения в рабочем проекте и управляют версиями при совместной работе над проектом.

Ядром технологии BIM является программный комплекс Autodesk Revit. Учитывая логику проектирования и этапы подготовки отдельных разделов проектной документации, рассмотрим последовательность использования программного обеспечения.

\section{Шаг первый}

Посредством Revit Architecture ocyществляется построение информационной модели объекта, основанной на 3D-визуализации с использованием блоков конструктивных элементов и установления взаимосвязи между ними. В результате формируется наглядная цифровая модель объекта, которую можно исследовать, анализировать и оценивать на любом этапе проектирования. Характеристика составных частей цифровой модели объекта включает в себя не только название конструктивных элементов, но и их структуру с указанием материалов, характеристик и стоимости, что позволяет в автоматическом режиме формировать спецификации. К информации, которую можно получить на основании информационной модели, относятся технико-экономические показатели, объёмно-планировочные решения, автоматическое построение фасадов и т.д. Данная модель является основой для работы на следующих этапах выполнения проектных работ (расчёты несущей способности конструкций, прокладка инженерных систем) и является основой для их взаимной увязки. Основным преимуществом данного вида технологий является то, что связь всех продуктов имеет двухсторонний характер, что позволяет в автоматическом режиме вносить изменения во все части проекта.

\section{Шаг второй}

Данная информационная модель передаётся в Revit Structure для конструкторского обоснования, опреде- 


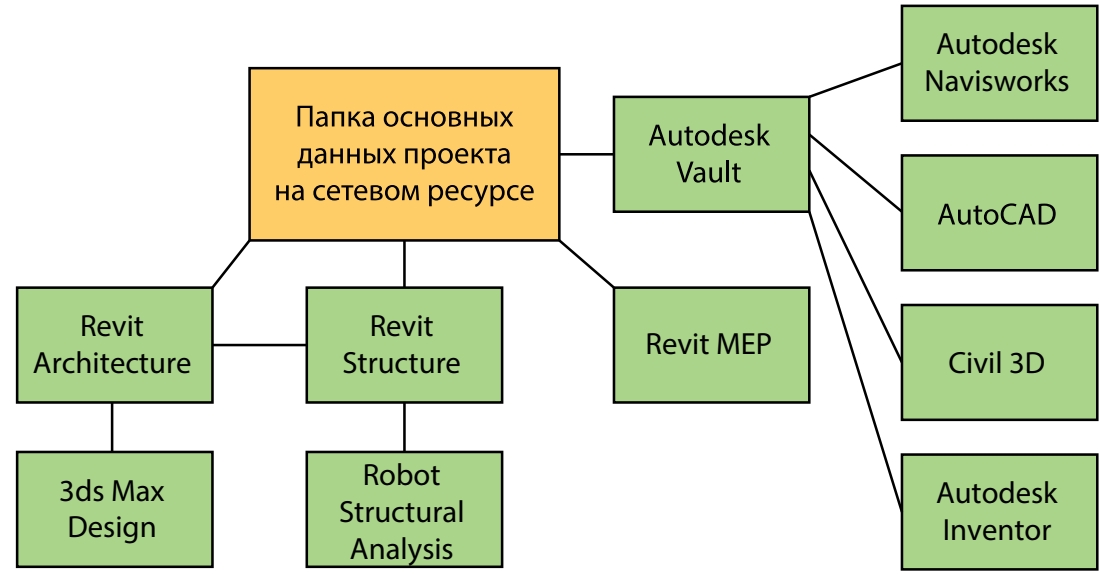

Puc. 1. Структура совместной работы в Revit с применением Autodesk Vault

ления несущих конструкций, формирования аналитической модели.

Полученная аналитическая модель передаётся в Autodesk Robot Structural Analysis для проведения расчётов конструктивных элементов. При проведении расчётов возможно моделирование различных нагрузок, в том числе динамических, сейсмических, ветровой, снеговой в разных комбинациях. В процессе расчётов есть возможность учесть нагрузку от движущихся механизмов, кранов и т.д. В результате подбирается сечение конструкций и их элементов, армирование железобетонных конструкций, проводится расчёт металлических каркасов и т.д. Формирование чертежей и спецификаций выполняется в автоматическом режиме в соответствии с действующей нормативной литературой. После этого модель передаётся в Revit Architecture и направляется смежникам для разработки инженерных систем.

\section{Шаг третий}

При помощи Revit MEP разрабатываются инженерные системы на базе динамических моделей с учётом характеристик режимов, потоков, расходов, подбора оборудования, расчёта освещённости, автоматической увязки всех систем, их пересечений и автоматического формирования чертежей, экспликации материалов и оборудования. Все мероприятия выполняются на базе 3D-модели, что значительно упрощает проектирование и снижает ошибки.

Параллельно с выполнением работ по проектированию инженерных систем, после расчёта конструктивных элементов, модель может быть передана в AutoCAD Structural Detailing для дальнейшей деталировки разделов КМ, КЖ, выполнения узлов и формирования отправочных чертежей.

После передачи всех инженерных разделов в Revit Architecture возможно проведение оценки энергетических и эксплуатационных характеристик объекта на весь срок его службы. В автоматическом режиме формируется энергетический паспорт объекта с расчётом потребления энергетических ресурсов по годам, месяцам.

\section{Шаг четвёртый}

Разработка схемы планировочной организации земельного участка. Данный раздел выполнялся при помощи Civil 3D. После подготовки раздела информация может быть передана обратно в Revit Architecture для подготовки материалов для визуализации.

\section{Пятый шаг}

Для координации процессов проектирования, обработки и наполнения разделов организации строительства и проекта производства строительных работ информационная модель передаётся в Autodesk Navisworks. Данный продукт позволяет при помощи 4D-визуализации проследить за процессом строительства с имитационным моделированием работы строительной техники.

\section{Шестой шаг}

Полученная информационная модель из Revit Architecture со всеми настройками по текстурам экстерьеру и генеральному плану, передаётся в Autodesk 3ds Max Design, где формируется окончательный вариант изображения, создаются видеоролики для демонстрации проектных идей.

Для оптимизации, автоматизации и синхронизации информационной модели работы с удалёнными участниками проекта Autodesk предлагает использовать продукт Autodesk Vault. Структура совместной работы Revit с приложением Autodesk Vault представлена на рисунке 1.

Работу с удалёнными пользователями и внутри проектной организации можно организовать и без использования Autodesk Vault, но в этом случае для актуализации данных необходимо будет в ручном режиме запускать Revit Architecture.

Как уже неоднократно отмечалось, комплекс программ Autodesk не учитывает некоторые особенности проектирования таких ЛО как автомобильные дороги. В частности, отсутствуют программные продукты, при помощи которых возможно проведение моделирования транспортных потоков. Группу продуктов Autodesk возможно применять при проектировании сооружений, которые могут входить в структуру автомобильной дороги (развязки, путепроводы, виадуки и т.д.).

Рассмотрим на другом примере реализацию ВIM-технологии, её логику и взаимосвязи. Программный комплекс Allplan был разработан немецкой компанией Nemetschek и предназначен, в том числе для проектирования автомобильных дорог.

Программный комплекс Allplan представляет собой единое инструментальное средство проектирования. Спектр его функциональных возможностей достаточно разнообразен и охватывает все этапы проектно-конструкторских работ: от эскизного проекта с оценкой вариантов прохождения трассы, грубой экономической оценки до детальной разработки проектной документации и подготовки демонстрационных материалов. Эта система соединяет в себе универсальный пользовательский интерфейс, необходимые средства компьютерной графики и формирования проектов на бумажных и электронных носителях.

Программный комплекс Allplan может использоваться как для организации показов и презентаций, 
подготовки и представлении строительно-технической документации, набросков, рисунков и произвольных моделей, так и для создания высококачественных изображений и фильмов. Кроме того, программный комплекс имеет функцию просмотра виртуальных моделей, проектируемых зданий и сооружений.

Работа с цифровой моделью здания позволяет управлять каждым отдельным элементом проекта, что представляет собой основное преимущество интегрированной системы проектирования. Allplan оперирует теми же объектами, которые используются непосредственно при строительстве: плиты перекрытия, лестницы, стропила, окна, арматура, венткороба и т.д. Модель здания формируется в соответствии с замыслом архитектора и в последовательности, соответствующей технологии строительства в трёх измерениях, а двумерные изображения, - эскизы, виды и разрезы, рабочие чертежи и спецификации, создаются и автоматически обновляются на основе 3D-модели.

Японская компания FORUM8 разработала программный комплекс VR-Design Studio, имеющий двухстороннюю связь с Allplan. Основной задачей комплекса является обучение вождению автомобиля и проработки действий в чрезвычайных ситуациях с использованием виртуальной реальности.

VR-Design Studio решает две задачи:

1. Максимально реалистично моделировать окружающий мир, создавая

Структурно программный комплекс условно можно разбить на две части: первая, относящаяся к модулю Allplan, связана с моделированием элементов конструкций зданий и сооружений, что является непосредственной реализацией ВIM-технологии. Вторая часть отвечает за моделирование транспортных, пешеходных потоков и их визуализацию.

AdvanceSteel/Concrete - блок моделирования металлических и железобетонных конструкций (связан с Allplan).

DesignBuilder - блок создания модели зданий, объектов электро-, водо-, теплоснабжения и экологии (связан c Allplan).

Engineer's Studio - блок для анализа нелинейного поведения структуры объектов капитального строительства (связан c Allplan).

Multiframe - блок для структурного анализа ОКС (связан с Allplan).

UC-1 и UC-Draw - блок проектирования зданий и сооружений различного назначения (жилые, промышленные здания и сооружения) (связан c Allplan и VR-Studio).

UC-1 vehicle trajectory, OSCADY/ TRANSYT, Micro Simulation - блоки моделирования транспортных потоков, расчёта состояний, имитации работы парковок. После работы передаёт результаты в VR-Studio.

Использование программного комплекса Allplan позволяет в очень короткие сроки оценить несколько вариантов прохождения трассы с укрупнённым подсчётом капитальных затрат. На последующих этапах

\section{Работа с цифровой моделью здания позволяет управлять каждым отдельным элементом проекта, что представляет собой основное преимущество интегрированной системы проектирования.}

и управляя различными природными и погодными условиями, такими как дождь, снег, ветер, освещение в различных фазах дня и географического положения на земле, тенями, временем суток.

2. Возможность использовать дополнительные технические средства, ресурсы и плагины, разработанные другими производителями. Это дополнительные программные модули или приборы. Например, лазерные сканеры, лидары и т.д. проектирования с минимальными временными потерями формирует комплект проектной документации автомобильной дороги.

Основным недостатком программного комплекса Allplan является то, что он не учитывает особенности нормативной базы Российской Федерации и невозможность определения энергетических и других эксплуатационных затрат.

Отечественный рынок САПР, которые могут использоваться для ре- ализации BIM-технологии и концепции поддержки жизненного цикла автомобильных дорог, представлен несколькими продуктами. С учётом задач, которые стоят перед проектировщиками, и соответствия основным принципам концепции поддержки жизненного цикла автомобильных дорог наибольший интерес представляет линейка продуктов, разработанная компанией из Томска «ИндорСофт».

Во-первых, это широкий спектр программного обеспечения, осуществляющий поддержку задач, начиная от проведения инженерных изысканий, проектирования и заканчивая эксплуатацией объекта.

Во-вторых, наибольший перечень функций на всех стадиях проектирования, которые являются базовыми для реализации концепции поддержки жизненного цикла. К таким функциям можно отнести: формирование 3D-модели непосредственно в процессе подготовки документации, возможности проверки, увязки отдельных частей проекта между собой, проверка нескольких проектных решений на стадии предпроектной проработки.

Тем не менее, анализ задач, которые могут быть решены при помощи продуктов «ИндорСофт», и функций, которые реализует тот или иной программный продукт, показал, что, на наш взгляд, некоторые направления проработаны не в полном объёме.

Необходимо решить вопросы, связанные с моделированием транспортных потоков, для проверки функционирования проектируемых объектов, расширить блок экономической оценки (в том числе и экспресс-метод) проектируемых объектов с учётом эксплуатационных затрат в рамках прогнозируемого срока эксплуатации и т.д.

Тем не менее, на наш взгляд, продукты компании «ИндорСофт» могут выступить основой для реализации концепции поддержки жизненного цикла объекта на основе информационного моделирования дорог. 드

Литература:

1. Бойков В.Н. САПР АД - перспективь развития // САПР и ГИС автомобильных дорог. 2013. №1(1). С. 6-9. 